\title{
Social facilitation of a previously learned response in normal, hypoglycemic, and alloxan diabetic rats
}

JAMES W. MCDANIEL, UNIVERSITY OF COLORADO SCHOOL OF MEDICINE SHELBY J. CLAYSON, UNIVERSITY OF MINNESOTA SCHOOL OF MEDICINE

The presence of a co-actor on performance of a well-learned T-maze response reduced behavior efficiency in terms of running time, but not accuracy. The effect interacts with blood glucose levels, Alloxan diabetic rats being relatively unaffected by pairing with another animal while normals and hypoglycemics showed inhibiting and facilitating effects respectively.

A meaningful analysis of the effects of the presence of another organism upon the learning of new responses and the performance of previously learned responses has recently been provided by Zajonc (1965) in a theoretical review of the topic of "social facilitation." Zajonc has considered both audience and co-actor effects, and has concluded with the hypothesis that the social facilitation effect lies in the enhancement of the dominant response to the situation, whatever it may be. This review of the somewhat contradictory evidence led to the inference, "It would appear that the emission of well-learned responses is facilitated by the presence of spectators, while the acquisition of new responses is impaired." This is said to be due to the predominance of incorrect responses in the early stages of learning, but the dominance of the correct well-learned response in later performance. Co-actor and audience effects are apparently identical in the enhancement of the emission of dominant responses. An attempt has been made by Zajonc to draw evidence together supporting the notion that increased arousal is responsible for the enhancement, and most of the diverse investigations reviewed would seem to support his inferences regarding both the origin and effect of this facilitation.

There have been very few studies of the effect of a co-actor on the performance of an already well-learned response, a response which, according to Zajonc (1965), should be facilitated by the presence of another organism. Dominant unc ondit ioned responses such as feeding, nest building, and exploratory behavior do seem to be enhanced by social facilitation. There is even less literature concerning the effects of blood glucose level on either learning or social facilitation.

The present investigation has paired animals already trained to a high level of performance in a $\mathrm{T}$-maze who were either normal, hypoglycemic or Alloxan diabetic. Two pair of rats of each combination of these three conditions $(\mathrm{N}=24)$ were then mun in the $\mathrm{T}$-maze to determine the interaction of social facilitation and blood glucose level. Ader, Kreutner, \& Jacobs (1963) have shown, using an open field test, that Alloxan diabetic rats when caged and reared individually or in groups, were more active and more emotional when group raised. Grouped Ss also had consistently higher blood glucose levels as well. Conceivably then, there is some interaction of blood glucose and social facilitation.

Ss were 24 albino rats (Holtzman). All were waterdeprived on a $16 \mathrm{hr}$. schedule. Ss had been trained to a level of $90 \%$ correct responses for two consecutive days in a $\mathrm{T}$-maze constructed of $3 / 4 \mathrm{in}$. plyboard and painted flat black. The maze had a stem 36 in. long with arms of 22 in. The start box measured 9 in. $x 9$ in. and each goal box was 10 in. $x 12$ in. Runways were 4 in. wide with 12 in. high walls. Retracing in the maze was prevented by the use of doors at each choice point, and the maze was rotated after each run. Ss were trained in the maze by first pre-training, placing in the maze five times a day for five days, followed on the sixth day by conditioning trials. Correct responses were reinforced by allowing animals to drink from a water tube in the goal box for $3 \mathrm{sec}$. Ten trials a day were given during original learning until criterion had been met.

Rats were made diabetic by injections of $5 \%$ Alloxan monohydrate $(120 \mathrm{mg} / \mathrm{kg})$ subcutaneously, and were considered to be diabetic if blood glucose level exceeded $200 \mathrm{mg} \%$ within 12-24 hr. following injection. Blood glucose levels in these Ss were thereafter held within normal limits $(\overline{\mathrm{X}}=119 \mathrm{mg} \%)$ with Isophane Insulin Suspension (NPH). Hypoglycemia was induced and maintained by daily injections of regular insulin (0.29$0.33 \mathrm{u}$ ), and mean blood glucose level was $48 \mathrm{mg} \%$ during the experimental period. Differences between normal and hypoglycemic $S s$ in mean blood glucose were significant $(t=8.79, p<.001)$. Differences between normal and diabetic controlled Ss were, however, not significant $(t=0.14)$. During original learning, a significant positive correlation resulted between mean running times and blood glucose level $(r=.39, p<.05)$, but there was no significant relation between mean number of correct responses and blood glucose. A

Table 1. Performance During Original Learning

\begin{tabular}{lcc} 
Subgroup & $\begin{array}{c}\bar{X} \text { No. trials } \\
\text { to criterion }\end{array}$ & $\begin{array}{c}\bar{X} \text { running } \\
\text { times }\end{array}$ \\
\hline Normal & 73 & 8.0 \\
Diabetic & 38 & 4.5 \\
Hypoglycemic & 145 & 14.1 \\
\hline
\end{tabular}


summary of the performance of the three subgroups in original learning is given in Table 1.

Differences between the three groups in both mean number of trials to reach criterion and mean running times were significant as tested by analysis of variance. Analysis of trials to criterion and running times yielded $F$ ratios of $10.57(\mathrm{df}=2 / 27, \mathrm{p}<.001)$ and $9.09(\mathrm{df}=2 / 27$, $p<.001)$, respectively.

Following the period of original learning, Ss were paired in the maze for four blocks of 10 trials using the same procedures and deprivation schedule as followed previously. Since the subgroups of animals differed significantly in their rates of original learning even though having been trained to the same level of response efficiency, differences due to various treatment combinations were expected.

Comparisons between the mean running times for correct responses for the last 40 trials of original learning with 40 trials of paired performance failed to indicate significance $(F=3.43, \mathrm{df}=1 / 36)$. There was, however, a significant effect due to the various combinations of subgroups $(F=6.18, d f=5 / 36, p<.001)$, and the interaction between subgroup combination and original vs. paired performance was also significant $(F=3.17, d f=5 / 36, p<.05)$. Table 2 indicates the discrepancies and their direction for each $\mathrm{S}$ in the six combinations between the mean running times for correct responses in original learning and paired performance with a base of 40 trials each. Running times were generally (but not significantly) increased during paired performance as compared to original learning. However, this effect was dependent upon the combination of Ss. The greatest effect came in pairings including hypoglycemic Ss, which for the normalhypoglycemic pairs increased running times sharply. Diabetic Ss were not so severely affected by pairing with a hypoglycemic animal, but then neither were they much affected by any pairing. Hypoglycemic Ss paired with each other produced the only condition in which running times were consistently improved by pairing in

Table 2, Discrepancy (Increase or Decrease)* in Running Times from Original Learning to Paired Performance in 24 Rats

\begin{tabular}{llll} 
& Normal & Diabetic & Hypoglycemic \\
\hline \multirow{3}{*}{ Normal } & +7.15 & +6.30 & +25.26 \\
& +1.36 & $+1.79 \mathrm{D}$ & $+58.66 \mathrm{H}$ \\
& +4.69 & +2.86 & +3.97 \\
& +3.52 & $+.30 \mathrm{D}$ & $+17.92 \mathrm{H}$ \\
& & & -.50 \\
& & +3.82 & $-3.66 \mathrm{H}$ \\
& & +.87 & +5.26 \\
Diabetic & +.32 & $+9.96 \mathrm{H}$ \\
& & -1.21 \\
& & -6.32 \\
Hypoglycemic & & -10.08 \\
& & -16.30 \\
\hline
\end{tabular}

* + indicates increased and - decreased running times in secs. the maze. Probably, this is due to the initial value of running time originally, which was quite low for this group, leaving greater room for improvement during paired performance. Normal and diabetic Ss were differentially affected by being paired with a hypoglycemic animal $(F=5.37$, df $=1 / 8, p<.05)$, diabetic Ss being relatively little affected, while normal Ss' running times suffered considerably from this pairing.

In general, the efficiency of response in terms of running speed is disrupted by the presence of another organism, both Ss having been conditioned to have the same dominant response to the situation. The frequency of correct responses did not suffer by pairing. Ss having been trained solitarily to a criterion of $90 \%$ correct responses on two consecutive days maintained this level of performance when paired with another animal. There was evidence, at least qualitatively, that the disruptive effect was a temporary one, and that behavioral efficiency tended to be restored after a few trials of experience in paired running.

Much of the effect of this study may be explained by the pairing of hypoglycemic Ss with other animals. The hypoglycemic animals were characteristically inactive, slow to learn originally, with extremely slow running times and high rate of non-response. This condition seemed to have an inhibiting effect upon the response efficiency of normal running mates.

Although no objective evidence can be offered at this time to support the following interpretation, inferentially these results may also be explained by metabolic differences in motivation with respect to water reinforcement for the controlled diabetic animals. They not only learned faster originally under identical deprivation and reinforcement conditions than the normal subgroup, but were less "distracted" from the goal during paired performance. Whereas excessive thirst is usually regarded as symptomatic of certain types of diabetes (diabetes insipidus and mellitus), it is conceivable that our results may have been due to metabolic factors influencing motivation. The inferior performance of hypoglycemic Ss in maze learning is, of course, due to metabolic factors; and this influenced their running mates. A complicated relationship between drive, metabolism, and social arousal is indicated by these results.

\section{References}

Ader, R., Kreutner, A., \& Jacobs, H. Social environment, emotionality, and Alloxan diabetes in the rat. Psychosom. Med., 1963, $25,60-68$.

Zajonc, R. Social facilitation. Science, 1965, 149, 269-274.

\section{Note}

1. This research was supported, in part, by a grant (RT-10) from the Vocational Rehabilitation Administration, U. S. Dept. HEW, and by a VRA graduate traineeship in physical therapy awarded by the American Physical Therapy Association to the junior author. The experiment was conducted according to the American Psychol. Assoc. statement of "Guiding Principles for the Humane Care and Use of Animals," December, 1962. 